Series A

I. MATHEMATICA

438

\title{
SYMMETRIZATION AND EXTREMAL RINGS IN SPACE
}

BY

G. D. ANDERSON

H E L S I N K I 1969

S U O M A L A I N E N T I E D EAKA T EM I A

doi:10.5186/aasfm.1969.438 
Communicated 11 October 1968 by Olli Lehto and K. I. Virtanen 


\section{Introduction}

Suppose that $f$ is a diffeomorphism of a 3 -space domain $\Omega$ onto $\Omega^{\prime}$. Then $f$ is locally affine; that is, if $P \varepsilon \Omega$ the differential mapping $d f(P)$ carries the unit ball onto an ellipsoid with axes of lengths $a \geq b \geq c$. The dilatation functions

$$
H_{I}(P, f)=\left[\frac{a b}{c^{2}}\right]^{\frac{1}{2}}, H_{0}(P, f)=\left[\frac{a^{2}}{b c}\right]^{\frac{1}{2}}
$$

measure how much infinitesimal balls are distorted, hence providing a natural measure of how much $f$ differs from being conformal at $P$. These functions are bounded below by 1 , and are 1 at a point $P$ if and only if $f$ is conformal there. We say that $f$ is quasiconformal if either, and hence both, of these dilatations is bounded above in $\Omega$.

One research goal in the study of quasiconformal mappings is to determine their distortion properties. This can be accomplished by assigning to each ring $R$ a modulus $\bmod R$ which is invariant under conformal (Möbius) transformations and which has the property that for each quasiconformal mapping $f$ there is a number $K=K(f), \quad 1 \leq K<\infty$, with

$$
\frac{1}{K} \bmod R \leq \bmod f(R) \leq K \bmod R \text {. }
$$

If one can show that among all rings with a certain geometric property a particular ring is extremal, that is, has the maximum modulus, then this fact can be used to determine distortion properties for quasiconformal mappings (Cf. [10], [11]).

It is comparatively easy to prove that certain plane rings are extremal, because one can employ conformal mappings [22]. Frequently it is also intuitively evident which rings in space ought to be extremal, but since the only conformal mappings in $E^{3}$ are the Möbius transformations (Cf. [11, $\S 29]$ ), the proofs there become more difficult. The only method so far

1) This research was supported in part by the National Science Foundation, Grant 18913, and by a Fellowship from the Institute of Science and Technology University of Michigan. A portion of the work is included in a Ph.D. thesis written under the direction of Professor F. W. Gehring at the University of Michigan. 
successful has been symmetrization. In $\S 2$ of this paper we define the Steiner and Schwarz symmetrizations of space rings and state theorems, proved in [2], that these processes do not decrease the moduli of space rings. These symmetrization theorems enable us to show in $\S 3$ that two given rings are extremal.

These extremal rings, designated by $R_{3, d}$ and $R_{3, s}$, may be described as follows. For fixed $a, 0<a<1$, the ring $R_{3, d}=R_{3, d}(a)$ consists of the unit ball minus the disk $|x| \leq a, x_{3}=0$, while $R_{3, s}=R_{3, s}(a)$ is obtained from the unit ball by omission of the slit $\left|x_{1}\right| \leq a, x_{2}=x_{3}=0$. In $\S 3$ we show that these rings have the following extremal properties. Let $R$ be a space ring consisting of the unit ball minus a continuum $C$. If the projection of $C$ on some diametral plane is at least $\pi a^{2}$ in area, then $\bmod R \leq \bmod R_{3, d}(a)$. If the diameter of $C$ is at least $2 a$, then $\bmod R \leq \bmod R_{3, s}(a)$. In the same section we show that if the complement of a space ring $R_{3}$ lies in a plane $\Pi$ and forms there a plane ring $R_{2}$, then point symmetrization in $\Pi$ induces an operation on $R_{3}$ which does not decrease $\bmod R_{3}$.

We continue the study of these extremal rings in $\S 4$. We first show that $\bmod R_{3, d} \leq \bmod R_{2}$, where now $R_{2}$ denotes the plane ring consisting of the unit disk $\left|x_{1}+i x_{2}\right|<1$ minus the central slit $\left|x_{1}\right| \leq a, x_{2}=0$. This, together with a reference to [9], completes the double inequality $\bmod R_{3, d} \leq \bmod R_{2} \leq \bmod R_{3, s}$. In $\S 5$ we obtain upper and lower bounds in terms of $a$ for $\bmod R_{3, d}(a)$ and $\bmod R_{3, s}(a)$. Our main tool is the use of inequalities for elliptic functions [3]; these have also proved useful in [1]. In $\S 6$ we use the bounds obtained in $\S 5$ to study the asymptotic behavior of the moduli of these rings as $a$ tends to 0 . In $\S 7$ we introduce a generalized notion of quasiconformality, together with some material on extremal lengths, and in the final two sections we employ this theory to investigate the behavior of $\bmod R_{3, d}$ and $\bmod R_{3, s}$ as $a$ tends to 1 . We discover that these moduli behave essentially like the modulus of the spherical annulus $a<|x|<1$ as $a$ tends to 0 . But as $a$ tends to 1 the asrmptotic behavior of these three rings is markedly different one from another.

\section{Symmetrization of space rings}

2.1. Space rings. $A$ space ring $R$ is a domain in $E^{3}$ whose complement consists of a bounded component $C_{0}$ and an unbounded component $C_{\mathbf{1}}$. The conformal capacity of $R$ is defined [16] as

$$
\operatorname{cap} R=\inf _{u}|\nabla u|_{R}^{3} d \omega \text {, }
$$


where the infimum is taken over all real-valued functions $u=u(x)$ which are continuously differentiable in $R$ and have boundary values 0 on $\partial C_{0}$ and 1 on $\partial C_{1}$.

Next, the modulus of $R$ is defined [11] as

$$
\bmod R=\left[\frac{4 \pi}{\operatorname{cap} R}\right]^{\frac{1}{2}}
$$

This is analogous to the modulus of a plane ring, usually defined by means of conformal mappings. The modulus is invariant under conformal (Möbius) transformations and satifies an inequality of the type (2) for each quasiconformal mapping $f$. If $R$ is the spherical annulus $r_{1}<|x|<r_{2}$, then the modulus of $R$ is $\log r_{2} / r_{1}$ [13].

2.2. Symmetrization methods. Symmetrization is a geometric operation invented by Jacob Steiner and developed by Pólya and Szegö [19]. Two well-known kinds of symmetrization in the plane are the Steiner and point symmetrizations. In this section we consider analogues of these in 3-space- known as the Steiner and Schwarz symmetrizations [19], respectively in which the corresponding plane symmetrization is performed in each plane normal to the $x_{3}$ axis.

If $R$ is a bounded space ring and $R^{\prime}$ is obtained from it by one of these symmetrizations then $\bmod R \leq \bmod R^{\prime}$. This inequality was proved by Gehring [13] for spherical and point symmetrization. The proofs for the Steiner and Schwarz symmetrizations, while embodying certain additional technical difficulties, follow the outline of Gehring's argument; proofs in detail are included in [2]. Similar results for radial symmetrization have been obtained by Pfaltzgraff [18].

2.3. Steiner symmetrization of rings. Let $G$ be a bounded open set in $E^{3}$. We define a second set $G^{*}$, called the Steiner symmetrization of $G$ with respect to the $x_{1} x_{2}$ plane, as follows: Let $L=L\left(x_{1}, x_{2}\right)$ denote the line in $E^{3}$ through $\left(x_{1}, x_{2}, 0\right)$ that is parallel to the $x_{3}$ axis. Then $L \cap G^{*}=\varnothing$ if and only if $L \cap G=\varnothing$. If $L \cap G \neq \varnothing$, then $L \cap G^{*}$ is an open segment of length $m_{1}(L \cap G)$ which is bisected by the $x_{1} x_{2}$ plane.

If $F$ is a bounded closed set in $E^{3}$, we define $F^{*}$ as above except in the second case, where we take $L \cap F^{*}$ to be a closed segment of length $m_{1}(L \cap F)$ which is bisected by the $x_{1} x_{2}$ plane. If $m_{1}(L \cap F)=0$, then $L \cap F^{*}$ is the single point $\left(x_{1}, x_{2}, 0\right)$.

If $G$ and $F$ are a bounded domain and a continuum, respectively, it is easily verified that $G^{*}$ and $F^{*}$ have the same properties. It is also easily shown that if $E$ is a bounded open or closed set, then $C\left(E^{*}\right)$ is 
connected. Hence if $R$ is a bounded ring in $E^{3}$ and $C_{0}$ and $C_{1}$ are the two components of $C(R)$ then the set

$$
R^{*}=\left(R \cup C_{0}\right)^{*}-C_{0}^{*}
$$

is a ring, and we define this to be the Steiner symmetrization of $R$. Then $R^{*}$ has the following extremal property [2].

Theorem 1. Let $R$ be any bounded ring in $E^{3}$, and let $R^{*}$ denote its Steiner symmetrization. Then $\bmod R \leq \bmod R^{*}$.

2.4. Schwarz symmetrization of rings. Let $G$ be a bounded open set in $E^{3}$. Then $G^{* *}$, the Schwarz symmetrization of $G$ with respect to the $x_{3}$ axis, is defined as follows. Let $\Pi=\Pi\left(x_{3}\right)$ denote the plane through $\left(0,0, x_{3}\right)$ that is normal to the $x_{3}$ axis. Then $I I \cap G^{* *}=\varnothing$ if and only if $\Pi \cap G=\varnothing$. If $\Pi \cap G \neq \varnothing$, we take $\Pi \cap G^{* *}$ to be an open disk of area $m_{2}(\Pi \cap G)$ with center on the $x_{3}$ axis.

If $F$ is a bounded closed set in $E^{3}$, we define $F^{* *}$ as above except in the second case, where we take $\Pi \cap F^{* *}$ to be a closed disk of area $m_{2}(I \cap F)$ with center on the $x_{3}$ axis. If $m_{2}\left(\Pi \cap F^{\prime}\right)=0$, then $\Pi \cap F^{* *}$ is the single point $\left(0,0, x_{3}\right)$.

If $R$ is a bounded ring in $E^{3}$ and $C_{0}$ and $C_{1}$ are the two components of $C(R)$, then it is easily verified that the set

$$
R^{* *}=\left(R \cup C_{0}\right)^{* *}-C_{0}^{* *}
$$

is a ring, and we define this to be the Schwarz symmetrization of $R$. It can be shown that $R^{* *}$ enjoys the following extremal property [2].

Theorem 2. Let $R$ be any bounded ring in $E^{3}$, and let $R^{* *}$ denote its Schwarz symmetrization. Then $\bmod R \leq \bmod R^{* * *}$.

\section{Extremal space rings}

3.1. Space rings with complement in a plane. An interesting type of ring $R_{3}$ in 3 -space is one for which both components of $C\left(R_{3}\right)$ lie in a plane $\Pi$, say $x_{3}=0$, and for which the configuration $R_{2}=\Pi \cap R_{3}$ is a plane ring.

If $R_{2}$ is a plane ring and $C$ is the bounded component of $C\left(R_{2}\right)$, then point symmetrization in the plane replaces $R_{2}$ by a circular annulus $R_{2}^{\prime}: r_{1}<\left|x_{1}+i x_{2}\right|<r_{2}$ with $m_{2}(C)=\pi r_{1}^{2}$ and $m_{2}(R \cup C)=\pi r_{2}^{2}$. It is known that $\bmod R_{2} \leq \bmod R_{2}^{\prime}$ (Cf. [6]). But this process of plane symmetrization also replaces $R_{3}$ by a new space ring $R_{3}^{\prime}$ with $C\left(R_{3}^{\prime}\right)=C\left(R_{2}^{\prime}\right)$, 
and we shall show that the plane symmetrization increases the space modulus also. In the proof of this result we shall need the following.

Lemma 1. Let $R_{3}$ be an unbounded space ring with nondegenerate boundary components $C_{0}$ and $C_{1}$. Given $\varepsilon>0$, there exists a bounded ring $R$ separating the components of $C\left(R_{3}\right)$ for which

$$
\bmod R_{3}<(1+\varepsilon)^{\frac{1}{2}} \bmod R .
$$

Proof. This proof assumes a certain familiarity with the terminology of [13]. Since the boundary components of $R_{3}$ are non-degenerate, it follows by [16] that cap $R_{3}>0$. Therefore there exists a simple admissible function $u[13, \S 7]$ for $R_{3}$ such that

$$
(1+\varepsilon) \operatorname{cap} R_{3}>\int_{R_{3}}|\nabla u|^{3} d \omega .
$$

Now let $E_{i}$ be the component of $\{x: u(x)=i\}$ which contains $C_{i}, i=0,1$. Since $u(x)=1$ for sufficiently large $|x|, C\left(E_{1}\right)$ must be bounded. Next, we see that $E_{0}$ and $E_{1}$ are disjoint continua. Hence by Lemma 3.5 of [14] there exists a ring $R$, with $C_{0}^{\prime}$ and $C_{1}^{\prime}$ as the components of $C(R)$, such that $\partial C_{i}^{\prime} \subset E_{i} \subset C_{i}^{\prime}$ for $i=0,1$. Because $R \subset C\left(E_{1}\right), R$ is bounded. Since $C_{i} \subset E_{i} \subset C_{i}^{\prime}, R$ separates the components of $C\left(R_{3}\right)$. Finally, since $\partial C_{i}^{\prime} \subset E_{i}, u=0$ on $\partial C_{0}^{\prime}$ and 1 on $\partial C_{1}^{\prime}$. Thus $u$ is an admissible function $[13, \S 3]$ for $R$ and hence

$$
\operatorname{cap} R \leq \int_{R}|\nabla u|^{3} d \omega \leq \int_{R_{3}}|\nabla u|^{3} d \omega<(1+\varepsilon) \operatorname{cap} R_{3},
$$

from which (4) follows.

Theorem 3. Let $R_{3}$ be a space ring such that both components of $C\left(R_{3}\right)$ lie in a plane $\Pi$ and determine a plane ring $R_{2}$ there. Let $R_{3}^{\prime}$ be the space ring obtained by point symmetrizing the plane ring $R_{2}$. Then $\bmod R_{3} \leq \bmod R_{3}^{\prime}$.

Proof. For convenience let $\Pi$ be the plane $x_{3}=0$. We may assume that $\bmod R_{3}<\infty$, for otherwise [16] shows that $\bmod R_{3}^{\prime}=\infty$ and there is nothing to prove.

Given $\varepsilon>0$, by Lemma 1 there exists a bounded ring $R$ separating the components of $C\left(R_{3}\right)$ for which (4) holds. Then by Theorem 2,

$$
\bmod R \leq \bmod R^{* *},
$$

where $R^{* *}$ is the Schwarz symmetrization of $R$ with respect to the $x_{3}$ axis. But since $R^{* *}$ separates the boundary components of $R_{3}^{\prime}$, Lemma 2 of [13] yields 


$$
\bmod R^{* *} \leq \bmod R_{3}^{\prime}
$$

Combining (4), (5), and (6) we have $(1+\varepsilon)^{-\frac{1}{2}} \bmod R_{3}<\bmod R_{3}^{\prime}$, from which the theorem follows when we let $\varepsilon$ approach zero.

3.2. Extremal rings $R_{3, d}$ and $R_{3, s}$. Now fix $a, 0<a<1$. Throughout the rest of this paper $R_{2}$ will denote the plane ring consisting of the unit disk minus the symmetric slit $\left|x_{1}\right| \leq a, x_{2}=0$. By $R_{3, d}$ and $R_{3, s}$ we shall mean the space rings obtained from $R_{2}$ by rotation about the $x_{2}$ and $x_{1}$ axes, respectively. The ring $R_{3, d}=R_{3, d}(a)$ consists of the unit ball minus the closed central disk $|x| \leq a, x_{3}=0$, while the ring $R_{3, s}=R_{3, s}(a)$ consists of the unit ball minus the slit $\left|x_{1}\right| \leq a, x_{2}=x_{3}=0$.

The ring $R_{3, d}$ is extremal in the following sense.

Theorem 4. Let $R$ be any space ring consisting of the unit ball minus a continuum $C$, and suppose that the projection of $C$ on some diametral plane $\Pi$ is at least $\pi a^{2}$ in area, $0<a<1$. Then $\bmod R \leq \bmod R_{3, d}(a)$.

Proof. For convenience let $I I$ be the plane $x_{3}=0$, and let $R^{*}$ be the Steiner symmetrization of $R$ with respect to $I I$. Then by Theorem 1 , $\bmod R \leq \bmod R^{*}$. Now replace the continuum $C$ by its projection on the $x_{1} x_{2}$ plane. This yields a new ring $R^{\prime}$, and $b y$ the monotoneity of the space modulus [13, Lemma 2] $\bmod R^{*} \leq \bmod R^{\prime}$. Finally, Schwarz symmetrization with respect to the $x_{3}$ axis replaces $R^{\prime}$ by a ring $R_{3, d}(b)$ for some $b, 0<a \leq b<1$. But then by Theorem 1 and monotoneity we have $\bmod R^{\prime} \leq \bmod R_{3, d}(b) \leq \bmod R_{3, d}(a)$, and the proof is complete.

The ring $R_{3, s}$ enjoys the following extremal property.

Theorem 5. Let $R$ be any space ring consisting of the unit ball minus a continuum $C$ whose diameter is at least $2 a, 0<a<1$. Then $\bmod R \leq \bmod R_{3, s}(a)$.

Proof. Let $P_{1}$ and $P_{2}$ be points of $C$ such that $\left|P_{1}-P_{2}\right|=2 b$, $b \geq a$. For convenience we may assume that $P_{1} P_{2}$ is parallel to the $x_{1}$ axis. The Schwarz symmetrization of $R$ with respect to the $x_{1}$ axis yields a new ring $R^{\prime}$ and, by Theorem $2, \bmod R \leq \bmod R^{\prime}$. This inequality follows also from Gehring's result on spherical symmetrization [13. Theorem 1] or the work of Sabat in [21].

Now the bounded component $C^{\prime}$ of $C\left(R^{\prime}\right)$ contains a segment $p \leq x_{1} \leq p+2 b, x_{2}=x_{3}=0$, and if $C^{\prime}$ is replaced by this segment a new ring $R^{\prime \prime}$ results such that $\bmod R^{\prime} \leq \bmod R^{\prime \prime}$. Finally, Steiner symmetrization of $R^{\prime \prime}$ with respect to the $x_{2} x_{3}$ plane rields $R_{3, s}(b)$ and, by Theorem 1 and monotoneity, $\bmod R^{\prime \prime} \leq \bmod R_{3, s}(b) \leq \bmod R_{3 . s}(a)$. 


\section{A double inequality}

In this section we begin our investigation of the properties of $\bmod R_{3, d}$ and $\bmod R_{3, s}$. Our first goal is the following result.

Theorem 6. Let $R_{2}$ be the plane ring consisting of the unit disk minus the slit $\left|x_{1}\right| \leq a, x_{2}=0$, and let $R_{3, d}$ and $R_{3, s}$ be the space rings obtained by rotating $R_{2}$ about the $x_{2}$ and $x_{1}$ axes, respectively. Then

$$
\bmod R_{3, d} \leq \bmod R_{2} \leq \bmod R_{3, s} .
$$

Proof. For the first inequality in (7) we shall obtain a diffeomorphism $f_{d}$ of a spherical annulus $A<|x|<1$ onto $R_{3, d}$. This mapping can be shown to have positive Jacobian $J(x)$ and to map each radius of $A<|x|<1$ onto a curve that is normal to the image of each surface $|x|=r, A<r<1$. Under these conditions it follows from a result in [9] that

$$
\int_{1}^{1} D_{1}\left(f_{d}, r\right) \frac{d r}{r} \leq \bmod R_{3, d} \leq \int_{A}^{1} D_{2}\left(f_{d}, r\right) \frac{d r}{r},
$$

where, for $A<r<1$,

$$
D_{1}\left(f_{d}, r\right)=\min _{x=r}\left[\left.\frac{N(x)^{3}}{J(x)}\right|^{\frac{1}{2}}, D_{2}\left(f_{d}, r\right)=\max _{x \mid=r}\left\lfloor\frac{N(x)^{3}}{J(x)}\right]^{\frac{1}{2}} .\right.
$$

Here $N(x)$ is the stretching normal to $\mid x^{\prime}=r$.

We shall then use some inequalities for elliptic functions to prove that $D_{2}\left(f_{d}, r\right) \leq 1$ and finally apply the second half of $(8)$ to achieve the first half of (7). The functional $D_{1}\left(f_{d}, r\right)$ and the first half of (8) will be needed in the proof of a theorem in $\S 5$. The second half of $(7)$ follows directly from an inequality obtained by Gehring in [9].

The plane annulus $A<x_{1}+i x_{2}<1$ is mapped conformally (See [5, p. 28] or [17, pp. $280-295])$ onto $R_{2}$ by the Jacobian elliptic sine function

$$
y_{1}+i y_{2}=f\left(x_{1}+i x_{2}\right)=k^{\frac{1}{2}} \operatorname{sn}(i(u+i v), k),
$$

where

$$
u+i v=\frac{2 K}{\pi} \log \frac{x_{1}+i x_{2}}{A}-i K, A=\exp \frac{-\pi K^{\prime}}{4 K}, k=a^{2}
$$

$\log \left(x_{1}+i x_{2}\right)$ denotes the principal branch of the logarithm, and 


$$
\begin{aligned}
& K=K(k)=\int_{0}^{1}\left[\left(1-t^{2}\right)\left(1-k^{2} t^{2}\right)\right]^{-\frac{1}{2}} d t, \\
& K^{\prime}=K\left(k^{\prime}\right), k^{\prime}=\left(1-k^{2}\right)^{\frac{1}{2}} .
\end{aligned}
$$

For our purpose it will be convenient to employ Jacobi's imaginary transformation $s n(i z, k)=i \operatorname{tn}\left(z, k^{\prime}\right) \quad([4, \mathrm{p} .37],[5, \#$ 125.02]) to rewrite (9) as

$$
y_{1}+i y_{2}=f\left(x_{1}+i x_{2}\right)=i k^{\frac{1}{2}} \operatorname{tn}\left(u+i v, k^{\prime}\right) .
$$

We define the space mapping $y=f_{d}(x)$ from $A<|x|<1$ onto $R_{3, d}$ by rotating $A<\left|x_{1}+i x_{2}\right|<1$ and $R_{2}$ about the $x_{2}$ and $y_{2}$ axes. That is, we let

$$
t+i y_{2}=f\left(s+i x_{2}\right), \varphi=\Theta,
$$

where $(s, \Theta)$ and $(t, \varphi)$ are polar coordinates in the $x_{1} x_{3}$ and $y_{1} y_{3}$ planes, respectively. It is easily verified that this mapping satisfies the hypotheses for (8) and that

$$
\begin{aligned}
& D_{1}\left(f_{d}, r\right)=\min _{\mid x_{1}+i x_{2}=r} \mid \frac{x_{1}}{y_{1}} f^{\prime}\left(x_{1}+i x_{2}\right)^{\frac{1}{2}}, \\
& D_{2}\left(f_{d}, r\right)=\left.\max _{\left|x_{1}+i x_{2}\right|=r} \frac{x_{1}}{y_{1}} f^{\prime}\left(x_{1}+i x_{2}\right)\right|^{\frac{1}{2}} .
\end{aligned}
$$

Now by (12) and the differentiation formula $\frac{d}{d z}(\operatorname{tn} z)=(d n z) /\left(c n^{2} z\right)$ $([4$, p. 9$],[5, \# 731.10])$ we have

$$
\left|f^{\prime}\right|=\frac{2 K}{\pi r} k^{\frac{1}{2}}\left|\frac{d n\left(u+i v, k^{\prime}\right)}{c n^{2}\left(u+i v, k^{\prime}\right)}\right|,
$$

while the addition theorems $([4$, p. 38], $[5, \# 125.01])$ give

$$
\left|f^{\prime}\right|=\frac{2 K}{\pi r} k^{\frac{1}{2}} \frac{\left(d^{2} C^{2} D^{2}+k^{4} s^{2} c^{2} S^{2}\right)^{\frac{1}{2}}\left(1-S^{2} d^{2}\right)}{c^{2} C^{2}+s^{2} d^{2} S^{2} D^{2}}
$$

and

$$
\begin{aligned}
& y_{1}=-k^{\frac{1}{2}} \operatorname{Im} \frac{s D-i c d S C}{c C+i s d S D}=k^{\frac{1}{2}} \frac{S d\left(c^{2} C^{2}+s^{2} D^{2}\right)}{c^{2} C^{2}+s^{2} d^{2} S^{2} D^{2}}, \\
& y_{2}=k^{\frac{1}{2}} \operatorname{Re} \frac{s D-i s d S C}{c C+i s d S D}=k^{\frac{1}{2}} \frac{s c C D\left(1-S^{2} d^{2}\right)}{c^{2} C^{2}+s^{2} d^{2} S^{2} D^{2}} .
\end{aligned}
$$

Here we have used the notation 
(17)

$$
\begin{aligned}
& s=s n\left(\frac{2 K}{\pi} \log \frac{r}{A}, k^{\prime}\right), c=d n\left(\frac{2 K}{\pi} \log \frac{r}{A}, k^{\prime}\right), d=d n\left(\frac{2 K}{\pi} \log \frac{r}{A}, k^{\prime}\right), \\
& S=s n\left(\frac{2 K}{\pi} \psi, k\right), C=c n\left(\frac{2 K}{\pi} \psi, k\right), D=d n\left(\frac{2 K}{\pi} \psi, k\right),
\end{aligned}
$$

where $\left(r, \frac{\pi}{2}-\psi\right)$ are polar coordinates in the $x_{1} x_{2}$ plane.

But using the identities $C^{2}=1-S^{2}, D^{2}=1-k^{2} S^{2}, c^{2}+s^{2}=1$, $c^{2}+k^{2} s^{2}=d^{2} \quad([4$, p. 9$],[5, \# 121.00])$ we achieve

$$
c^{2} C^{2}+s^{2} D^{2}=1-S^{2} d^{2} .
$$

Hence from $(15),(16),(18)$, and the fact that $x_{1}=r \sin \psi$, there results

$$
\frac{x_{1}}{y_{1}} f^{\prime}=\frac{2 K}{\pi}|\sin \psi|\left[\frac{C^{2} D^{2}}{s^{2}}+k^{\prime 4} \frac{s^{2} c^{2}}{d^{2}}\right]^{\frac{1}{2}} .
$$

We wish to show that $D_{2}\left(f_{d}, r\right) \leq 1, A<r<1$. For this it is sufficient to prove that sup $D_{2}\left(f_{d}, r\right) \leq 1$. According to Landen's Transformation $([4$, p. 72$],[5, \# 163.01])$,

$$
\left.\frac{s c}{d}=(1+k)^{-1} s n \mid(1+k) \frac{2 K}{\pi} \log \frac{r}{A}, \frac{1-k}{1+k}\right\rfloor .
$$

Since $s n(u, k)$ is maximum when $u=K([5, \# 121.02],[24, p .499])$ and because $K((1-k) /(1+k))=(1+k) K^{\prime} / 2 \quad([4$, p. 72$],[5, \# 164,02])$ we see that $s c / d$ has its maximum value $(1+k)^{-1}$ when $\log r / A=\pi K^{\prime} / 4 K$ $=\log 1 / A$, that is, when $r=1$. Thus

$$
\sup _{r} D_{2}\left(f_{d}, r\right)^{2}=\sup _{\psi} \frac{2 K}{\pi}(\sin \psi)\left[\frac{C^{2} D^{2}}{S^{2}}+(1-k)^{2}\right]^{\frac{1}{2}} .
$$

Now by use of the identities $C^{2}=1-S^{2}, D^{2}=1-k^{2} S^{2}$ $([5, \# 121.00],[24$, p. 493$])$ we see that

$$
C^{2} D^{2}+(1-k)^{2} S^{2}=\left(1-k S^{2}\right)^{2},
$$

so that $(21)$ reduces to

$$
\sup _{r} D_{2}\left(f_{d}, r\right)^{2}=\sup _{\psi} \frac{2 K}{\pi} \frac{(\sin \psi)}{S}\left(1-k S^{2}\right) .
$$


We have shown in [3] that the expression on the right of (23) is bounded above by 1 . Hence $D_{2}\left(f_{d}, r\right) \leq 1$ for $A<r<1$, and by (8) we have

$$
\bmod R_{3, d} \leq \int_{A}^{1} D_{2}\left(f_{d}, r\right) \frac{d r}{r} \leq \log \frac{1}{A}=\bmod R_{2}
$$

concluding the proof of the theorem.

\section{Bounds for the moduli of $\boldsymbol{R}_{3, d}$ and $\boldsymbol{R}_{3, s}$}

Next, using elliptic integrals, we obtain upper and lower bounds in terms of $a$ for $\bmod R_{3, d}(a)$.

Theorem 7. For each $a, 0<a<1$,

$\frac{1}{2}\left(\frac{\pi}{2 K}\right)^{\frac{1}{2}}\left[K\left(\left[\frac{1+k^{\prime}}{2}\right]^{\frac{1}{2}}\right)-K\left(\left\lfloor\frac{1-k^{\prime}}{2}\right]^{\frac{1}{2}}\right)\right] \leq \bmod R_{3, d}(a) \leq \frac{\pi K^{\prime}}{4 K}$,

where $k=a^{2}, k^{\prime}=\left(1-a^{4}\right)^{\frac{1}{2}}$, and $K=K(k)$ and $K^{\prime}=K\left(k^{\prime}\right)$ denote the elliptic integrals in (11).

Proof. Since $\bmod R_{2}=\log 1 / A=\pi K^{\prime} / 4 K$, the upper bound follows immediately from Theorem 6 .

To obtain the lower bound we apply the left side of (8) to $\bmod R_{3, d}(a)$. Thus by (14) and (19) we must determine

$$
D_{1}\left(f_{d}, r\right)^{2}=\min _{x_{1}+i x_{2}=r} \frac{2 K}{\pi}|\sin \psi|\left[\frac{C^{2} D^{2}}{S^{2}}+k^{\prime 4} \frac{s^{2} c^{2}}{d^{2}}\right]^{\frac{1}{2}},
$$

where $S, C, D, s, c$, and $d$ have the meanings assigned in (17). We assert that the minimum is achieved, for each $r$, when $\psi=\frac{\pi}{2}$. To see this, we use (20), (22), and the identity $s n^{2} z+c n^{2} z=1$ ([5, \#121.00], [24, p. 493]) to rewrite (25) as

$$
D_{1}\left(f_{d}, r\right)^{2}=\frac{2 K}{\pi} \min _{\psi,}|\sin \psi|\left[\frac{\left(1-k S^{2}\right)^{2}}{S^{2}}-(1-k)^{2} c n^{2}\left(z, k_{1}{ }^{\prime}\right)\right]^{\frac{1}{2}},
$$

where $z=(1+k) \frac{2 K}{\pi} \log \frac{r}{A}$ and $k_{1}{ }^{\prime}=(1-k) /(1+k)$. But since $|\sin \psi|$ is maximum when $\psi=\pi / 2$, and since by $(5)$ of [3] the expression $(\sin \psi)\left(1-k S^{2}\right) / S$ achieves its minimum $1-k$ when $\psi=\pi / 2$, we conclude that the minimum in (26) occurs when $\psi=\pi / 2$. 
Hence by (25),

$$
D_{1}\left(f_{d}, r\right)^{2}=\frac{2 K}{\pi} k^{2} \frac{s c}{d}
$$

and combining this with (8) we obtain the inequality

$$
\bmod R_{3, d} \geq k^{\prime}\left(\frac{2 K}{\pi}\right)^{\frac{1}{2}} \int_{A}^{1}\left(\frac{s c}{d}\right)^{\frac{1}{2}} \frac{d r}{r}
$$

To evaluate the integral in (27) we first apply the half angle formulas $\left([5, \# 124.02],\left[15\right.\right.$, p. 120]) and the identity $d n^{2} z+k^{2} s n^{2} z=1$ $([5, \# 121.00],[24$, p. 493]) to write

$$
\frac{s c}{d}=\frac{1-d n\left(\frac{4 K}{\pi} \log \frac{r}{A}, k^{\prime}\right)}{k^{\prime 2} \operatorname{sn}\left(\frac{4 K}{\pi} \log \frac{r}{A}, k^{\prime}\right)} .
$$

Now making the change of variables

$$
\text { sn }\left(\frac{4 K}{\pi} \log \frac{r}{A}, k^{\prime}\right)=\frac{t^{2}}{2-t^{2}}
$$

we have

$$
\left[\frac{1-d n\left(\frac{4 K}{\pi} \log \frac{r}{A}, k^{\prime}\right)}{k^{\prime 2} s n\left(\frac{4 K}{\pi} \log \frac{r}{A}, k^{\prime}\right)}\right]^{\frac{1}{2}}=\frac{\left[1-\frac{1-k^{\prime}}{2} t^{2}\right]^{\frac{1}{2}}-\left[1-\frac{1+k^{\prime}}{2} t^{2}\right]^{\frac{1}{2}}}{k^{\prime} t}
$$

and

$$
\frac{d r}{r}=\frac{\pi}{4 K}\left[\left(1-t^{2}\right)\left(1-\frac{1-k^{\prime}}{2} t^{2}\right)\left(1-\frac{1+k^{\prime}}{2} t^{2}\right)\right]^{-\frac{1}{2}} t d t
$$

When $r$ is $A, 1$ then $t$ is 0,1 , respectively, and by means of (28), $(29)$, and (30) we may reduce (27) to

$$
\begin{aligned}
\bmod R_{3, d} \geq & \frac{1}{2}\left(\frac{\pi}{2 K}\right)^{\frac{1}{2}}\left[\int_{0}^{1}\left[\left(1-t^{2}\right)\left(1-\frac{1+k^{\prime}}{2} t^{2}\right)\right]^{-\frac{1}{2}} d t\right. \\
& \left.\int_{0}^{1}\left[\left(1-t^{2}\right)\left(\begin{array}{cccc}
1 & 1-k^{\prime} \\
2 & t^{2}
\end{array}\right)\right]^{-\frac{1}{2}} d t\right] .
\end{aligned}
$$


Finally, consulting the definition (11) of the elliptic integral $K=K(k)$ we arrive at the first half of (24), and the theorem is proved.

Our methods also yield the following bounds for $\bmod R_{3, s}(a)$.

Theorem 8. For each $a, 0<a<1$,

$$
\frac{\pi K^{\prime}}{4 K} \leq \bmod R_{3, s}(a) \leq \frac{1}{2}\left(\frac{\pi}{2 K}\right)^{\frac{1}{2}}\left[K\left(\left[\frac{1+k^{\prime}}{2}\right]^{\frac{1}{2}}\right)+K\left(\left[\frac{1-k^{\prime}}{2}\right]^{\frac{1}{2}}\right)\right]
$$

where $k=a^{2}, k^{\prime}=\left(1-a^{4}\right)^{\frac{1}{2}}$, and $K=K(k)$ and $K^{\prime}=K\left(k^{\prime}\right)$ denote the elliptic integrals in (11).

Proof. The lower bound follows directly from Theorem 6 .

To obtain the upper bound we employ a technique introduced in the proof of Theorem 6 . Let $f$ be the conformal mapping (12) of the plane annulus $A<\left|x_{1}+i x_{2}\right|<1$ onto $R_{2}$, and let $y=f_{s}(x)$ be the space mapping of $A<|x|<1$ onto $R_{3, s}$ obtained from $f$ by rotating $a<\left|x_{1}+i x_{2}\right|<1$ and $R_{2}$ about the $x_{1}$ and $y_{1}$ axes. Then $f_{s}$ is a diffeomorphism satisfying (8), where

$$
\begin{aligned}
& D_{1}\left(f_{s}, r\right)=\left.\min _{x_{1}+i x_{2}-\cdots r} \frac{x_{2}}{y_{2}} f^{\prime}\left(x_{1}+i x_{2}\right)\right|^{\frac{1}{2}} \\
& D_{2}\left(f_{s}, r\right)=\max _{x_{1}+i x_{2}-r} \frac{x_{2}}{y_{2}} f^{\prime}\left(x_{1}+i x_{2}\right)^{\frac{1}{2}} .
\end{aligned}
$$

We now apply the right hand side of (8) to $\bmod R_{3, s}$. Thus by (15) and (16) we must determine

$$
D_{2}\left(f_{s}, r\right)^{2}=\max _{x_{1}+i x_{2} ; \cdots r} \frac{2 K}{\pi}|\cos \psi|\left\lfloor k^{4} \frac{S^{2}}{C^{2} D^{2}}+\frac{d^{2}}{s^{2} c^{2}}\right]^{\frac{1}{2}},
$$

where $S, C, D, s, c$, and $d$ are the functions defined in (17).

We assert that this maximum is achieved, for each fixed $r$, when $\psi=0$. Because of the special values $s n 0=0$, cn $0=d n 0=1 \quad([4, \mathrm{p} .9]$, $[5, \# 122.01])$, this is equivalent to the assertion that

$$
\left(\cos ^{2} \psi\right)\left[k^{\prime 4} \frac{S^{2}}{C^{2} D^{2}}+\frac{d^{2}}{s^{2} c^{2}}\right] \leq \frac{d^{2}}{s^{2} c^{2}}
$$

for all $\psi$.

By a simple rearrangement and use of the identity $\cos ^{2} \psi=1-\sin ^{2} \psi$, this is reduced to the claim that

$$
k^{\prime 4} \frac{s^{2} c^{2}}{d^{2}} \leq \frac{C^{2} D^{2}}{S^{2}} \tan ^{2} \psi .
$$


Elsewhere [3] we have shown that

$$
\frac{2 k^{\prime} K}{\pi} \leq \frac{c n\left(\frac{2 K}{\pi} \psi, k\right)}{s n\left(\frac{2 K}{\pi} \psi, k\right)} \tan \psi
$$

for all real $\psi$. Since $D \geq k^{\prime}([5, \# 121.02],[24$, pp. 493, 499]) it follows from (34) that

$$
\frac{C D}{S} \tan \psi \geq \frac{2 k^{\prime 2} K}{\pi} .
$$

Then, comparing (35) with (33), we see that to show that the maximum in (32) occurs when $\psi=0$ it is sufficient to prove

$$
\frac{s c}{d} \leq \frac{2 K}{\pi}
$$

We proved earlier, after $(20)$, that $s c / d \leq(1+k)^{-1}$. Thus (36) is implied by

$$
1 \leq(1+k) \frac{2 K}{\pi}
$$

The latter inequality, however, is trivial, since $k \geq 0$ and $K \geq \pi / 2$.

We conclude that (33) is valid, and that the maximum in (32) occurs for $\psi=0$. Hence

$$
D_{2}\left(f_{s}, r\right)^{2}=\frac{2 K}{\pi} \frac{d}{s c} .
$$

By virtue of (8), this means that

$$
\bmod R_{3, s} \leq\left(\frac{2 K}{\tau}\right)^{\frac{1}{2}} \int_{1}^{1}\left(\frac{d}{s c}\right)^{\frac{1}{2}} \frac{d r}{r} .
$$

Evaluation of this integral by the same change of variables used in the proof of Theorem 8 then yields the upper bound in (31).

\section{Behavior of $\bmod R_{3, d}$ and $\bmod R_{3, s}$ for small a}

Let us turn now to the study of the asymptotic behavior of these extremal rings. We first obtain, as applications of the theorems of the preceding section, a pair of asymptotic formulas for the moduli as $a$ tends to 0 . 
Theorem 9. As a function of $a,\left(\bmod R_{3, d}(a)-\log 1 / a\right)$ is monotone decreasing in the interval $0<a<1$, and

$$
\lim _{a \rightarrow 0}\left(\bmod R_{3, d}(a)-\log 1 / a\right)=c_{1}
$$

where $0.254 \ldots=\frac{3}{2} \log 2-\frac{\pi}{4} \leq c_{1} \leq \log 2=0.693 \ldots$.

Proof. For let $0<a^{\prime}<a<1$. If $R$ is the image of $R_{3, d}\left(a^{\prime}\right)$ under the conformal mapping $y=\left(a / a^{\prime}\right) x$, then $\bmod R_{3, d}\left(a^{\prime}\right)=\bmod R$. Since $R_{3, d}(a)$ and $1<|x|<a / a^{\prime}$ are two disjoint rings separating the boundary components of $R$, we see from Lemma 2 of [13] that

$$
\bmod R_{3, d}(a)+\log a / a^{\prime} \leq \bmod R=\bmod R_{3, d}\left(a^{\prime}\right),
$$

from which the monotoneity follows (Cf. Lemma 6 in [13]).

To obtain the upper bound we make use of Theorem $6,(10)$, and the limit $\lim _{k \rightarrow 0} k^{2} \exp \left(\pi K^{\prime} / K\right)=16 \quad$ (Cf. [5, \# 112.04, \#901.00], [7, p. 88]) to conclude that

$$
\begin{aligned}
\lim _{a \rightarrow 0}\left(\bmod R_{3, d}-\log 1 / a\right) & \leq \lim _{a \rightarrow 0}\left(\bmod R_{2}-\log 1 / a\right) \\
& =\lim _{k \rightarrow 0}\left|\frac{\pi K^{\prime}}{4 K}+\log k^{\frac{1}{2}}\right|=\log 2 .
\end{aligned}
$$

To obtain the lower bound we use Theorem $\tau$ and the fact that $K(0)=\pi / 2$ to obtain

$$
\lim _{a \rightarrow 0}\left(\bmod R_{3, d}-\log 1 / a\right) \geq \frac{1}{2} \lim _{k \rightarrow 0}\left[\left[\log k+K\left(\left[\frac{1+k^{\prime}}{2}\right]^{\frac{1}{2}}\right)\right]-\frac{\pi}{4} .\right.
$$

Then by means of the $\operatorname{limit} \lim _{k \rightarrow 0}\left(K^{\prime}-\log 4 / k\right)=0([5, \# 112.01]$, $\left[24\right.$, p. 521]) we may reduce the right side of $(38)$ to $\frac{1}{2} \log 8-\pi / 4$, which is the lower bound in the theorem.

Theorem 10. As a function of $a,\left(\bmod R_{3 . s}(a)-\log 1 / a\right)$ is monotone decreasing in the interval $0<a<1$, and

$$
\lim _{a \rightarrow 0}\left(\bmod R_{3, s}(a)-\log 1 / a\right)=c_{2},
$$

where $0.693 \ldots=\log 2 \leq c_{2} \leq \frac{3}{2} \log 2+\frac{\pi}{4}=1.82 \ldots$.

Proof. The argument for monotoneity is the same as that given in Theorem 9. To obtain the lower bound we make use of Theorem 6 as in (37) to conclude that 


$$
\lim _{a \rightarrow 0}\left(\bmod R_{3, s}-\log 1 / a\right) \geq \lim _{a \rightarrow 0}\left(\bmod R_{2}-\log 1 / a\right)=\log 2 .
$$

Finally, use of Theorem $\varepsilon$, together with a proof similar to that given for the lower bound in Theorem 9, gives

$$
\lim _{a \rightarrow 0}\left(\bmod R_{3,-}-\log 1 / a\right) \leq \frac{3}{2} \log 2+\frac{\pi}{4},
$$

which is the upper bound in the theorem.

We remark that the bounds in Theorem 10 were obtained by Gehring $([9, \S 9],[13, \S 21])$, the upper bound by a quite different method.

\section{Quasiconformality and extremal lengths}

Before we can study the asymptotic behavior of the moduli of $R_{3, d}$ and $R_{3, s}$ as a tends to 1 , we need some additional tools. We begin by returning to (1), the dilatation functions $H_{1}(P, f)$ and $H_{0}(P, f)$ defined in $\S 1$. If $f$ is a diffeomorphism of a 3-space domain $\Omega$ onto $\Omega^{\prime}$, then the functionals

$$
K_{I}(f)=\sup _{P_{\varepsilon} \Omega} H_{1}(P, f), K_{0}(f)=\sup _{P_{\varepsilon} \Omega} H_{0}(P, f)
$$

are known as the inner and outer dilatations of $f$, respectively. These dilatations are simultaneously infinite or finite. If both are finite, then $f$ is called a quasiconformal mapping.

These definitions may be generalized, by means of the theory of rings, to include an arbitrary homeomorphism $f$ of $\Omega$ onto $\Omega^{\prime}$. The inner and outer dilatations of a homeomorphism $f$ are defined as

$$
K_{I}(f)=\sup _{R} \frac{\bmod R}{\bmod f(R)}, \quad K_{0}(f)=\sup _{R} \frac{\bmod f(R)}{\bmod R},
$$

where the suprema are taken over all bounded rings $R$ with $\bar{R} \subset \Omega$ for which $\bmod R$ and $\bmod f(R)$ are not both infinite. If one of these dilatations is finite the other is also (Cf. (1.10) in [14]) and $f$ is said to be a quasiconformal mapping. In case $f$ is a diffeomorphism, this definition reduces to the one previously given [14, Lemma 1.1]. If $f$ is a homeomorphism of a ring $R$ onto a ring $R^{\prime}$, then it follows from Lemma 1 and (40) that

$$
K_{I}(f) \geq \frac{\bmod R}{\bmod R^{\prime}}, \quad K_{0}(f) \geq \frac{\bmod R^{\prime}}{\bmod R} .
$$

Next, we shall need a result from the theory of extremal lengths. Let $\Gamma$ be a family of ares in $E^{3}$, and let $F(T)$ denote the family of density functions $\varrho$ which are nonnegative and Borel measurable in $E^{3}$ and for which 


$$
\int_{\gamma} \varrho d s \geq 1
$$

for each $\operatorname{arc} \gamma \varepsilon \Gamma$. Here the integral is taken with respect to linear measure [20] if $\gamma$ is not locally rectifiable. Then following Väisälä [23] we define the modulus $M(\Gamma)$ of the family $\Gamma$ as

$$
M(\Gamma)=\inf _{\varrho \in F(\Gamma)} \int_{E^{3}} \varrho^{3} d \omega .
$$

(See also [8] and [14]). If $R$ is a space ring and $T$ is the family of ares which join the components of $\partial R$ in $R$, then by Theorem 1 of [9],

$$
\operatorname{cap} R=M(T) \text {. }
$$

\section{Behavior of $\bmod \mathbf{R}_{3, d}$ as a tends to 1}

Theorem 11. The modulus of $R_{3 . d}(a)$ has the following order as a tends to 1:

$$
\limsup _{a \rightarrow 1}(1-a)^{-\frac{1}{2}} \bmod R_{3, d}(a) \leq \frac{\pi}{2}=1.57 \ldots
$$

and

$$
\lim _{a \rightarrow 1} \inf (1-a)^{-\frac{1}{2}} \bmod R_{3, d}(a) \geq 0.65 \ldots .
$$

Proof. To obtain the upper bound (45) let $\Gamma_{1}$ be the family of arcs in $R_{3, d}$ joining the components of $\partial R_{3, d}$, and let $I$ be the subfamily of circular arcs $\gamma$ which are normal to the boundary components. Since $\Gamma_{1} \supset \Gamma$ we have $F\left(\Gamma_{1}\right) \subset F(\Gamma)$ and, by (43), $M\left(\Gamma_{1}\right) \geq M(\Gamma)$. We will prove that

$$
M(T) \geq \frac{32}{\pi} \frac{a^{2}}{1-a^{2}} .
$$

Then, using (43), (44), and (47), we will have

$$
\operatorname{cap} R_{3, d}=M\left(\Gamma_{1}\right) \geq M(\Gamma) \geq \frac{32}{\pi} \frac{a^{2}}{1-a^{2}} .
$$

By definition (3) of the modulus, this is equivalent to

$$
\left(\bmod R_{3, d}(a)\right)^{2} \leq \frac{\pi^{2}}{8} \frac{1-a^{2}}{a^{2}},
$$

from which (45) follows directly. 
To prove (47) let $\Omega$ be the intersection of the plane quadrant $x_{1}>0$, $x_{2}>0, x_{3}=0$ with the union of the curves in $\Gamma$ considered as point sets, and let $\Omega^{\prime}$ be the image of $\Omega$ under

$$
y_{1}+i y_{2}=f\left(x_{1}+i x_{2}\right)=\frac{1+\left(x_{1}+i x_{2}\right)}{1-\left(x_{1}+i x_{2}\right)} .
$$

Then $\Omega^{\prime}$ is the quarter annulus $1<\left|y_{1}+i y_{2}\right|<b=(1+a) /(1-a)$, $y_{1}>0, y_{2}>0, y_{3}=0$. If $g$ is the inverse of $f$, then it is easy to see that

$$
\left|g^{\prime}\left(y_{1}+i y_{2}\right)\right|=\frac{2}{\left|y_{1}+i y_{2}+1\right|^{2}}, x_{1}=\frac{\left|y_{1}+i y_{2}\right|^{2}-1}{\left|y_{1}+i y_{2}+1\right|^{2}} .
$$

Let $\varrho \varepsilon F(T)$. Then for each $\gamma \in \Gamma$ in $\Omega$ we have, by virtue of (42),

$$
\left.1 \leq \mid \int_{\gamma} \varrho d s\right]^{3}=\left|\int_{\gamma^{\prime}} \varrho^{\prime}\right| g^{\prime}\left|d s^{\prime}\right|^{3} \text {, }
$$

where $\varrho=\varrho\left(x_{1}+i x_{2}\right), \varrho^{\prime}=\varrho^{\prime}\left(y_{1}+i y_{2}\right)=\varrho\left(g\left(y_{1}+i y_{2}\right)\right)$, and $\gamma^{\prime}=f(\gamma)$ is a quarter circle $r=r_{0}, 0<\varphi<\pi / 2$, for some fixed $r_{0}, 1<r_{0}<b$. Then an application of Hölder's inequality to (49) yields

$$
1 \leq\left[\int_{\gamma^{\prime}} \varrho^{\prime 3} x_{1}\left|g^{\prime}\right|^{2} d s^{\prime}\right]\left[\int_{\gamma^{\prime}}\left|g^{\prime \frac{1}{2}} x_{1}^{-\frac{1}{2}} d s^{\prime}\right|^{2} .\right.
$$

Using (48) we may reduce (50) to

$$
\int_{\gamma^{\prime}} \varrho^{\prime 3} x_{1} \mid g^{\prime 2} d s^{\prime} \geq \frac{2}{\pi^{2}} \frac{r^{2}-1}{r^{2}},
$$

where $(r, \varphi)$ are polar coordinates in the $y_{1}+i y_{2}$ plane.

Next, we see that

$$
\int_{E^{3}} \varrho^{3} d \omega \geq 2 \int_{i}^{2 \cdot \pi}\left(\int_{\Omega} \varrho^{3} x_{1} d \sigma\right) d \Theta=4 \pi \int_{\Omega^{\prime}} \varrho^{\prime 3} x_{1}\left|g^{\prime}\right|^{2} d \sigma^{\prime} .
$$

But using (51) and the fact that $b=(1+a) /(1-a)$ we determine that

$$
\int_{\Omega^{\prime}} \varrho^{\prime 3} x_{1}\left|g^{\prime}\right|^{2} d \sigma^{\prime} \geq \frac{8}{\pi^{2}} \frac{a^{2}}{1-a^{2}} .
$$

Hence (52) gives the estimate

$$
\int_{E^{3}} \varrho^{3} d \omega \geq \frac{32}{\pi} \frac{a^{2}}{1-a^{2}}
$$

from which (47) follows when we take the infimum over all $\varrho \varepsilon F(\Gamma)$. 
Next we obtain the lower bound (46). For this, let $\left(r, \Theta, x_{2}\right)$ be cylindrical coordinates in $E_{3}$ and define

$$
u(x)=\left\{\begin{array}{l}
\left|x_{2}\right| /(1-r) \text { if }\left|x_{2}\right| \leq 1-r, 0 \leq r \leq a, \\
{\left[(r-a)^{2}+x_{2}^{2}\right]^{\frac{1}{2}} /(1-a) \text { if } \quad(r-a)^{2}+x_{2}^{2} \leq(1-a)^{2}, a \leq r \leq 1,} \\
1 \text { elsewhere. }
\end{array}\right.
$$

Then $u$ is admissible for $R_{3, d}(a)[13, \S 3]$, and an elementary integration gives

$$
\int_{\mathrm{R}_{3, d}(a)}|\nabla u|^{3} d \omega=\pi(4 p+\pi) \frac{a}{1-a}-4 \pi p \log \frac{1}{1-a}+\frac{4 \pi}{3},
$$

where $p=[7 \sqrt{2}+\log (7+5 \sqrt{2})] / 8$. Then, appealing to Lemma 1 in [13] we have

$$
\limsup _{a \rightarrow 1}(1-a) \operatorname{cap} R_{3, s}(a) \leq \pi(4 p+\pi),
$$

from which, because of (3), (46) follows immediately.

\section{Behavior of $\bmod \boldsymbol{R}_{3, s}$ as a tends to 1}

We conclude this paper by proving the following asymptotic formula for $\bmod R_{3, s}$.

Theorem 12. As a function of $a,\left[\log \frac{1+a}{1-a}\right]^{\frac{1}{2}} \bmod R_{3, s}(a)$ is monotone increasing in the interval $0<a<1$, and

$$
\lim _{a \rightarrow 1}\left\lfloor\log \frac{1+a}{1-a}\right]^{\frac{1}{2}} \bmod R_{3, s}(a)=c_{3},
$$

where $1.03 \ldots \leq c_{3} \leq q$,

$$
q=\int_{0}^{\pi / 2}(\sin t)^{-\frac{1}{2}} d t=2^{\frac{1}{2}} K\left(\left(\frac{1}{2}\right)^{\frac{1}{2}}\right)=2.62 \ldots
$$

Proof. We first prove the asserted monotoneity. There exists a Möbius transformation carrying $R_{3, s}(a)$ conformally onto the space ring $R(b)$ consisting of the half space $x_{2}>0$ minus the slit $1 \leq x_{2} \leq b, x_{1}=x_{3}=0$, where

$$
b=\left[\frac{1+a}{1-a}\right]^{2}
$$


The mapping

$$
g(x)=x|x|^{p-1}, \quad p=\left(\log b^{\prime}\right) /(\log b)
$$

is a diffeomorphism of $R(b)$ onto $R\left(b^{\prime}\right)$. Assuming for definiteness that $1<b^{\prime}<b$, we have $0<p<1$. Applying (39) and (41) it is easily seen that

$$
K_{0}(g)=p^{-\frac{1}{2}}=\left[\frac{\log b}{\log b^{\prime}}\right]^{\frac{1}{2}} \geq \frac{\bmod R\left(b^{\prime}\right)}{\bmod R(b)},
$$

from which it follows that $\left.(\log b)^{\frac{1}{2}} \bmod R_{,}^{\prime} \partial\right)$ is a monotone increasing function of $b$. Since by (54) $a$ and $b$ increase together, this shows that the expression in the theorem increases monotonically with $a$ as asserted.

To obtain the upper bound in the theorem let $E$ be the slit $1 \leq x_{2} \leq b$, $x_{1}=x_{3}=0$ and $I^{\prime}$ the family of arcs $\gamma^{\prime}$ in $1<|x|<b, \quad x_{2}>0$ which join $E$ to the plane annulus $1<\left|x_{1}+i x_{3}\right|<b, x_{2}=0$. Then Lemma 3.8 of [14] gives

$$
M\left(\Gamma^{\prime}\right)=\frac{2 \pi}{q^{2}} \log b,
$$

where $q$ is the elliptic integral in (53).

Now let $\Gamma$ be the family of arcs $\gamma$ joining the boundary components of $R(b)$ in $R(b)$. It follows from (44) and (55) that

$$
\operatorname{cap} R(b)=M(\Gamma) \geq M\left(\Gamma^{\prime}\right)=\frac{2 \pi}{q^{2}} \log b,
$$

and combining this with the fact that cap $R_{3, s}=$ cap $R(b)$, one arrives at

$$
\left(\bmod R_{3, s}\right)^{2}=\frac{4 \pi}{\operatorname{cap} R_{3, s}} \leq \frac{2 q^{2}}{\log b}=\frac{q^{2}}{\log \frac{1+a}{1-a}} .
$$

The upper bound in the theorem follows from (56) and the monotoneity already proved.

To obtain the lower bound we use the same method as for $(46)$ in $\S 8$. Let $\left(r, \Theta, x_{1}\right)$ be cylindrical coordinates in $E_{3}$, and define $u(x)=\left\{\begin{array}{l}r /\left(1-\left|x_{1}\right|\right) \text { if } 0 \leq r \leq 1-\left|x_{1},\right| x_{1} \mid \leq a \\ {\left[\left(\left|x_{1}\right|-a\right)^{2}+r^{2}\right]^{\frac{1}{2}} /(1-a) \text { if }\left(\left|x_{1}\right|-a\right)^{2}+r^{2} \leq(1-a)^{2}, a \leq\left|x_{1}\right| \leq 1,} \\ 1 \text { elsewhere. }\end{array}\right.$ Then $u$ is admissible for $R_{3, s}[13, \S 3]$, and an elementary integration gives 


$$
\int_{R_{3}, s}|\nabla u|^{3} d \omega=\frac{4 \pi(4 \sqrt{2}-1)}{5} \log \frac{1}{1-a}+\frac{2 \pi}{3} .
$$

Then, appealing again to Lemma 1 in [13] we have

$$
\limsup _{a \rightarrow 1} \frac{\operatorname{cap} R_{3, s}(a)}{\log \frac{1+a}{1-a}} \leq \frac{4 \pi(4 \sqrt{2}-1)}{5},
$$

from which, in view of (3), the lower bound in the theorem follows.

Remark. By point symmetrization in space [13, Theorem 2] one can verify that the spherical annular ring $R_{3, b}(a), 0<a<1$, consisting of the unit ball minus the closed ball $|x| \leq a$ is extremal in the following sense. Let $R$ be any space ring consisting of the unit ball minus a continuum $C$, and suppose $m_{3}(C) \geq 4 \pi a^{3} / 3$. Then $\bmod R \leq \bmod R_{3, b}(a)$.

Next, since $\bmod R_{3, b}(a)=\log 1 / a$, it is easily checked that as a function of $a$,

$$
\frac{1+a}{1-a} \bmod R_{3, b}(a)
$$

is monotone decreasing in the interval $0<a<1$ and that (57) has limit 2 as $a$ tends to 1 .

The pattern exhibited by (57) and Theorems 11 and 12 indicates how strongly influenced is the asymptotic behavior of these extremal rings, as $a$ tends to 1 , by the dimension of the central set omitted from the unit ball in the formation of the ring. Theorems 9 and 10 in this paper show, however, that all three of these rings have the same order as $a$ tends to 0 , so that for small $a$ the modulus is much less sensitive to the dimension of the bounded component of the complement. One naturally wonders if a pattern similar to this prevails for the analogous extremal rings in higher dimensions.

\section{Michigan State University}

East Lansing, Michigan, USA 


\section{References}

[1] Anderson, G. D.: The coefficients of quasiconformality of ellipsoids. - Ann. Acad. Sci. Fenn. Ser. A I 411 (1967), 1-14.

[2] -- - Extremal rings and quasiconformal mappings in 3-space. - Ph.D. Dissertation, University of Michigan, 1965.

[3] - -- Inequalities for elliptic functions. - Amer. Math. Monthly 74 (1967).

[4] Bowman, F.: Introduction to Elliptic Functions with Applications. - Dover, New York, 1961.

[5] Byrd, P. F., and M. D. Friedman: Handbook of Elliptic Integrals for Engineers and Physicists. - Springer, Berlin, 1954.

[6] Carleman, T.: Über ein Minimalproblem der mathematischen Physik. - Math. Z. 1 (1918), $208-212$.

[7] Enneper, A.: Elliptische Functionen, Theorie und Geschichte. - Louis Nebert, Halle a. S., 1890.

[8] Fuglede, B.: Extremal length and functional completion. - Acta Math. 98 (1957), $171-219$.

[9] Gehring, F. W.: Extremal length definitions for the conformal capacity of rings in space. - Mich. Math. J. 9. (1962), 137-150.

[10] -"- Quasiconformal mappings in space. - Bull. Amer. Math. Soc. 69 (1963), $146-164$.

[11] -》- Rings and quasiconformal mappings in space. - Proc. Nat. Acad. Sci. U.S.A., 47 (1961), $98-105$.

[12] - - Rings and quasiconformal mappings in space. - Trans. Amer. Math. Soc. $103(1962), 353-393$.

[13] -- - Symmetrization of rings in space. - Trans. Amer. Math. Soc., 101 (1961), $499-519$.

[14] - -- and J. VÄISÄLÄ.: The coefficients of quasiconformality of domains in space. - Acta Math. 114 (1965), 1-70.

[15] Greenhild, A. G.: The Applications of Elliptic Functions. - Dover, New York, 1959.

[16] Loewner, C.: On the conformal capacity in space. - J. Math. Mech. 8 (1959), $411-414$.

[17] Nehari, Z.: Conformal Mapping. - McGraw-Hill, New York, 1952.

[18] Pfaltzgraff, J. A.: Radial symmetrization and capacities in space. - Duke J. Math. 35 (1968).

[19] Pólya, G., and G. Szegö.: Isoperimetric Inequalities in Mathematical Physics. - Ann. of Math. Studies No. 27, Princeton, 1951.

[20] Saks, S.: Theory of the Integral. - Hafner, New York, 1937.

[21] ŠABAT, B. V.: On the theory of quasiconformal mappings in space. - Soviet Math. 1 (1960), $730-733$. 
A. I. 438

[22] TexchмüLleR, O.: Untersuchungen über konforme und quasikonforme Abbildung. - Deutsche Math. 3 (1938), $621-678$.

[23] VÄIs⿱̈亡̈̈, J.: On quasiconformal mappings in space. - Ann. Acad. Sci. Fenn. A I 298 (1961), $1-36$.

[24] Whittaker, E. T., and G. N. Watson: Modern Analysis, Cambridge, 1958. 